\title{
EVOLUÇÃO DOS SAIS NO SOLO IRRIGADO COM ÁGUA SALINA E APLICAÇÃO DE BIOFERTILIZANTE
}

\section{SALTS EVOLUTION IN SOIL IRRIGATED WITH SALINE WATER AND BIOFERTILIZER APPLICATION}

\begin{abstract}
Robson Alexsandro de Sousa ${ }^{1}$; Cherlyson Cunha de Medeiros ${ }^{2}$; Claudia Larissa Bernardo da Silva ${ }^{3}$; Claudio Bernardo da Silva ${ }^{4}$; Claudivan Feitosa de Lacerda ${ }^{5}$
\end{abstract}

DOI: https://doi.org/10.31692/978-65-991061-7-0.339-344

\section{INTRODUÇÃO}

Várias alternativas para minorar os efeitos da água salina, tanto no solo quanto nas plantas, vêm sendo testadas dentre elas a aplicação de biofertilizante aparece como forma de reativar a atividade microbiológica do solo, prejudicada pelo conteúdo elevado de sais da água de irrigação (VAN HORN et al., 2014). A ação positiva dos biofertilizantes em atenuarem parcialmente os efeitos da salinidade da água de irrigação na formação de mudas de algumas culturas como goiabeira (CAVALCANTE et al., 2010), devido a presença de substâncias húmicas, contidas nos insumos orgânicos, proporcionarem uma maior regulação osmótica entre a raiz e a solução do solo, entretanto, a existe a falta de estudos sobre o efeito no solo quando se utiliza o biofertilizante, concomitantemente, a água salina.

Portanto, o objetivo deste trabalho foi avaliar a salinidade do solo através da condutividade elétrica e a porcentagem de saturação de sódio, quando foram aplicadas diferentes doses de biofertilizante e irrigação com águas de crescentes níveis de sais num solo cultivado com sorgo cv BRS Ponta Negra.

\section{FUNDAMENTAÇÃO TEÓRICA}

A região do Nordeste brasileiro, principalmente na sua porção de semiárido, apresenta-se com escassez de água, na qual a razão entre disponibilidade e a demanda pelos recursos hídricos é uma das mais preocupantes do país (DIAS et al., 2012). Nesta região, caracterizada por elevada evapotranspiração, em torno de $2000 \mathrm{~mm} \mathrm{ano}^{-1}$, e baixos índices pluviométricos, os solos tendem a apresentar alta concentração de bases trocáveis decorrente do incipiente processo de lixiviação, indicando a princípio, solos de alta fertilidade. No entanto, em áreas irrigadas, pelo intenso processo de solubilização de minerais há acúmulo de

\footnotetext{
${ }^{1}$ Doutor em Engenharia Agrícola, EAJ-UFRN, E-mail: rasousaufrn@gmail.com

2 Acadêmico em Engenharia Agronômica, Escola Agrícola de Jundiaí-UFRN, E-mail: medeiros-if@ hotmail.com

3 Acadêmica em Engenharia Agronômica, Escola Agrícola de Jundiaí-UFRN, E-mail: larissaclaudia9@gmail.com

${ }^{4}$ Acadêmico em Zootecnia, Escola Agrícola de Jundiaí-UFRN, E-mail: claudio171292@ hotmail.com

${ }^{5}$ Doutor em Fisiologia Vegetal, Universidade Federal do Ceará, E-mail: cfeitosa@ufc.br
} 
íons, que quando precipitam, originam solos com acúmulo de sais, limitando sua fertilidade e a produtividade das culturas (SANTOS et al., 2010).

A aplicação de biofertilizantes desempenha um papel vital na manutenção da fertilidade do solo a longo prazo (MISHRA et al., 2013) mantendo o ambiente do solo rico em todos os tipos de micro e macro nutrientes via fixação biológica de nitrogênio, fosfato e potássio, solubilização ou mineralização, com liberação de substâncias que regulam o crescimento das plantas e a biodegradação da matéria orgânica no solo, sendo utilizando em condições de estresse salino como forma de atenuar os efeitos deletérios da salinidade (BADAR et al., 2015).

\section{METODOLOGIA}

O experimento foi conduzido em casa de vegetação localizada na Escola Agrícola de Jundiaí - Unidade Acadêmica Especializada em Ciências Agrárias, pertencente a Universidade Federal do Rio Grande do Norte, localizada no município de Macaíba -RN. Utilizou-se a cultura do sorgo cv. BRS Ponta Negra, classificada na categoria forrageiro de pequeno. O delineamento experimental utilizado foi o inteiramente casualizado com cinco repetições no esquema fatorial 4 x 3, totalizando doze tratamentos. Foram avaliadas quatro doses do biofertilizante comercial $\left(75,150,225\right.$ e $\left.300 \mathrm{~L} \mathrm{ha}^{-1}\right)$, e as plantas de sorgo cv. BRS Ponta Negra foram irrigadas com quatro níveis de salinidade da água $(0,2 ; 2,0 ; 4,0$ e 6,0 dS m ${ }^{1}$ ). O delineamento experimental utilizado foi o inteiramente casualizado com quatro repetições no esquema fatorial 4 x 4, totalizando 16 tratamentos.

Na Tabela 1, observa-se a composição química das águas utilizadas para a irrigação no experimento. Para o preparo das soluções salinas, foram utilizados os sais de $\mathrm{NaCl}$, $\mathrm{CaCl}_{2} \cdot 2 \mathrm{H}_{2} \mathrm{O}$ e $\mathrm{MgCl}_{2} \cdot 6 \mathrm{H}_{2} \mathrm{O}$ dissolvidos em água de açude, na proporção de 7:2:1, obedecendo-se à relação entre a condutividade elétrica da água de irrigação (CEa) e sua concentração $\left(\right.$ mmolc $\left.\mathrm{L}^{-1}=\mathrm{CE} \times 10\right)$.

Tabela 1. Composição química das águas de irrigação usadas no experimento.

\begin{tabular}{|c|c|c|c|c|c|c|c|c|c|c|}
\hline \multirow{2}{*}{ Água } & $\mathrm{Ca}^{2+}$ & $\mathrm{Mg}^{2+}$ & $\mathrm{Na}^{+}$ & $\mathrm{K}^{+}$ & $\mathrm{Cl}^{-}$ & $\mathrm{CO}_{3}{ }^{2-}$ & $\mathrm{HCO}_{3}^{-}$ & \multirow{2}{*}{$\mathrm{pH}$} & \multirow{2}{*}{$\begin{array}{c}\mathrm{CEa} \\
\left(\mathrm{dS} \mathrm{m}^{-1}\right)\end{array}$} & \multirow{2}{*}{ RAS } \\
\hline & \multicolumn{7}{|c|}{$\mathrm{mmol}_{\mathrm{C}} \mathrm{L}^{-1}$} & & & \\
\hline S0 & 0,15 & 0,22 & 0,85 & 0,20 & 1,12 & 0,00 & 0,42 & 7,0 & 0,20 & 1,11 \\
\hline S1 & 0,50 & 0,50 & 21,35 & 0,15 & 19,81 & 0,00 & 0,33 & 6,4 & 2,00 & 15,69 \\
\hline S2 & 0,70 & 0,30 & 41,39 & 0,16 & 38,50 & 0,00 & 0,28 & 6,2 & 4,00 & 26,85 \\
\hline S3 & 0,50 & 0,50 & 56,35 & 0,15 & 54,15 & 0,00 & 0,24 & 6,2 & 6,00 & 37,03 \\
\hline
\end{tabular}

Fonte: Laboratório de Análises de Solo, Água e Planta - EMPARN. CEa = condutividade elétrica da água de irrigação; RAS = relação de adsorção de sódio. $\mathrm{S} 0$ = água de açude do Bebo; $\mathrm{S} 1$ = solução salina 1; S2 = solução salina 2; S3 = solução salina 3.

O turno de rega foi diário. A quantidade de água aplicada no experimento foi estimada 
com o objetivo de o solo alcançar a sua capacidade de campo e o excesso de água percolasse adicionando-se uma fração de lixiviação de 15\%, aproximadamente. Até o desbaste, para a irrigação, utilizou-se água de Açude do Bebo (S0). Para a instalação do experimento, colocouse aproximadamente $23 \mathrm{~kg}$ de solo arenoso (Tabela 2) em vasos plásticos de $32 \mathrm{~cm}$ de diâmetro na base maior e $24 \mathrm{~cm}$ de diâmetro na base menor e altura $34 \mathrm{~cm}$, perfurados na face inferior.

Tabela 2. Atributos químicos e classificação textural do solo utilizado no experimento.

\begin{tabular}{|l|c|c|c|c|c|c|c|c|c|c|c|c|c|c|}
\hline $\mathrm{Ca}^{2+}$ & $\mathrm{Mg}^{2+}$ & $\mathrm{Na}^{+}$ & $\mathrm{K}^{+}$ & $\mathrm{H}^{+}+\mathrm{Al}^{3+}$ & $\mathrm{Al}^{3+}$ & $\mathrm{SB}$ & $\mathrm{t}$ & $\mathrm{P}$ & $\mathrm{pH}$ & $\mathrm{CE}_{\mathrm{es}}$ & $\mathrm{PST}$ & $\mathrm{V}$ & $\mathrm{Dg}$ & $\mathrm{T}$ \\
\hline \multicolumn{8}{|c|}{$\mathrm{cmol}_{\mathrm{k}} \mathrm{kg}^{-1}$} & & $\left(\mathrm{mg} \mathrm{dm}^{-3}\right)$ & & $\left(\mathrm{dS} \mathrm{m}^{-1}\right)$ & $(\%)$ & $\left(\mathrm{g} \mathrm{cm}^{-3}\right)$ & \\
\hline 0,8 & 0,7 & 0,05 & 0,13 & 1,82 & 0,65 & 1,7 & 3,5 & 1,31 & 5,0 & 0,1 & 1,0 & 49 & 1,46 & Areia \\
\hline
\end{tabular}

Fonte: Laboratório de Solos e Água - DCS/CCA/UFC. SB = soma de bases; $\mathrm{t}=$ capacidade de troca catiônica efetiva; $\mathrm{pH}=$ pH em água $(1: 2,5) ; \mathrm{CE}_{\mathrm{es}}=$ condutividade elétrica do extrato de saturação; PST = porcentagem de sódio trocável; $\mathrm{V}=$ saturação por bases; $\mathrm{Dg}=$ densidade global; $\mathrm{T}=$ textura

As aplicações das doses do biofertilizante (Tabela 3), tiveram início aos dez dias após a semeadura. O biofertilizante foi diluído em água não salina, colocando-se em cada vaso as doses de $0,75,1,5,2,25$ e 3,00 mL/vaso/vez, correspondentes às doses de biofertilizante, 75, 150,225 e $300 \mathrm{~L} \mathrm{ha}^{-1}$, respectivamente.

Tabela 3 - Composição química do biofertilizante líquido concentrado utilizado no experimento.

\begin{tabular}{|c|c|c|c|c|c|c|c|c|c|c|}
\hline \multirow{2}{*}{$\begin{array}{l}\text { Biofertilizante } \\
\quad\left(\mathrm{L} \mathrm{ha}^{-1}\right)\end{array}$} & $\mathrm{Ca}^{2+}$ & $\mathrm{Mg}^{2+}$ & $\mathrm{Na}^{+}$ & $\mathrm{K}^{+}$ & $\mathrm{Cl}^{-}$ & $\mathrm{CO}_{3}^{-}$ & $\mathrm{HCO}_{3}$ & $\mathrm{pH}$ & \multirow{2}{*}{ 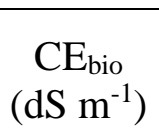 } & \multirow{2}{*}{$\mathrm{RAS}_{\text {bio }}$} \\
\hline & \multicolumn{8}{|c|}{$\mathrm{mmol}_{\mathrm{c}} \mathrm{L}^{-1}$} & & \\
\hline 75 & 34,00 & 14,00 & 0,46 & 0,07 & 25,00 & 0,00 & 1,20 & 4,40 & 4,04 & 0,10 \\
\hline 150 & 63,00 & 24,30 & 0,58 & 0,11 & 25,00 & 0,00 & 1,70 & 4,30 & 7,26 & 0,10 \\
\hline 225 & 91,50 & 31,80 & 0,81 & 0,16 & 25,00 & 0,00 & 1,90 & 4,40 & 9,78 & 0,10 \\
\hline 300 & 114,80 & 46,70 & 1,10 & 0,21 & 30,00 & 0,00 & 2,00 & 4,40 & 12,51 & 0,10 \\
\hline
\end{tabular}

Fonte: Laboratório de Fertilidade do Solo e Nutrição de Plantas - DCS/UFERSA. CE $_{\mathrm{B}}=$ condutividade elétrica do biofertilizante concentrado. $\mathrm{RAS}_{\mathrm{B}}=$ relação de adsorção de sódio do biofertilizante.

Após a coleta das plantas do experimento, aos 60 dias, retirou-se amostras de solo de cada vaso na profundidade de $20 \mathrm{~cm}$, nas cinco repetições de cada tratamento, sendo homogeneizadas formando uma amostra composta por tratamento, em seguida foram acondicionadas em sacos plásticos correspondente a cada tratamento, condutividade elétrica do extrato de saturação e porcentagem de sódio trocável no solo. As análises foram realizadas no Laboratório de Análises de Solo, Água e Planta, pertencente a Empresa de Pesquisa Agropecuária do Rio Grande do Norte (EMPARN).

Os resultados das variáveis foram submetidos à análise de variância e as médias comparadas pelo teste de Tukey com $\mathrm{p}<0,05$ (comparação das doses de biofertilizante) utilizando-se o programa ASSISTAT 7.6 Beta. A análise de regressão foi empregada para a 
avaliação dos efeitos da salinidade da água de irrigação e da interação, quando significativa.

\section{RESULTADOS E DISCUSSÕES}

Na figura $1 \mathrm{~A}$ verifica-se que os valores da condutividade elétrica do extrato de saturação são muito superiores ao encontrado no início do experimento (CEes $=0,10 \mathrm{dS} \mathrm{\textrm {m } ^ { - 1 }}$ ), sendo atribuído, esse resultado, ao aumento da salinidade da água de irrigação. Verifica-se, ainda, que o aumento das doses de biofertilizante também provocou elevação da CEes do solo, podendo ser explicado pelo valor alto da CE desse insumo (Tabela 3), no entanto, a contribuição do biofertilizante para a elevação da salinidade do solo apresentou uma variação muito pequena, pois segundo Ouni et al. (2014) a aplicação de matéria orgânica e, em consequência, a distribuição das substâncias húmicas diminuem no solo o $\mathrm{Na}^{+}$, a $\mathrm{CE}$ e o pH, provavelmente, devido às altas fontes de $\mathrm{Ca}^{+2}, \mathrm{Mg}^{+2}$ e $\mathrm{K}^{+}$, o que é evidenciado na composição do biofertilizante utilizado neste trabalho (Tabela 3), entretanto esse fato não foi observado neste trabalho pois houve aumento de forma linear quando se aplicou as diferentes doses de biofertilizantes.

Figura 1 - Valores médios de condutividade elétrica do extrato de saturação (A) e porcentagem de saturação de sódio (B) do solo em função da salinidade da água de irrigação e aplicação de biofertilizante. Fonte: Própia
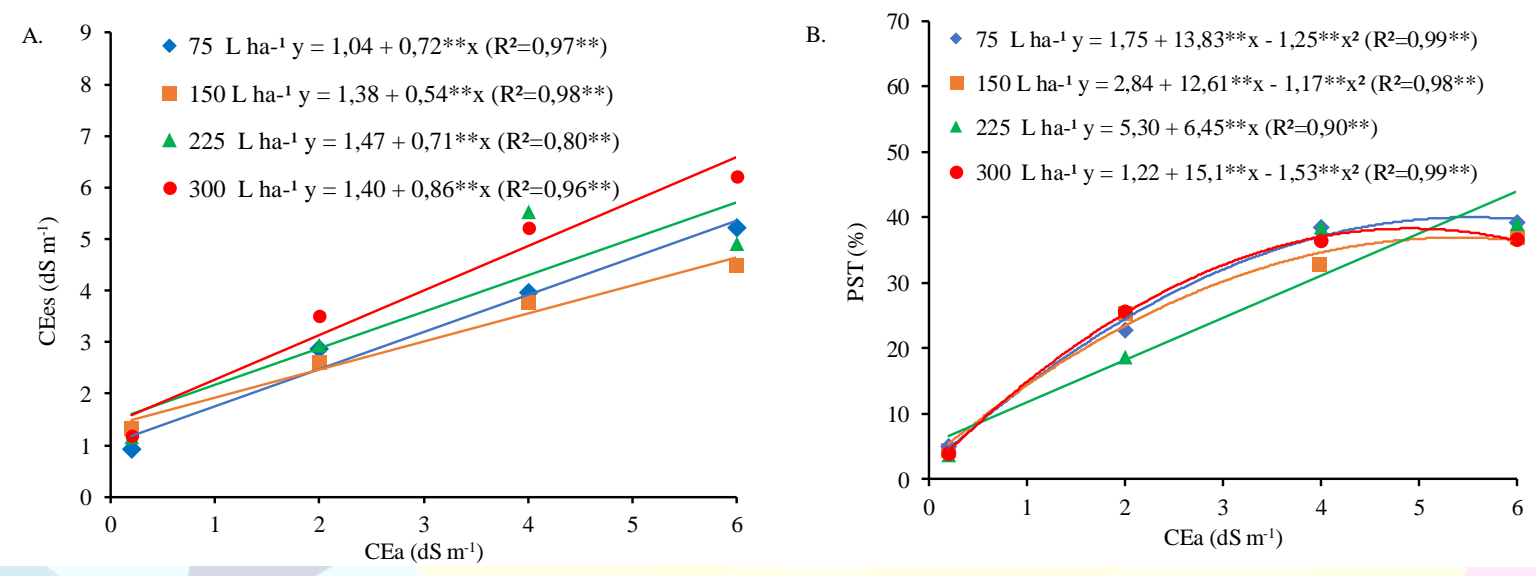

Resultado semelhante ao encontrado por Lima Neto et al. (2015) ao constatar que a condutividade elétrica do extrato de saturação do solo aumentou linearmente em função da salinidade da água, independentemente da aplicação dos biofertilizantes, mas sempre com maior intensidade nos tratamentos com os insumos orgânicos aplicados ao solo na forma líquida.

Os valores da porcentagem de sódio trocável (PST), figura 1A, apresentou valores superiores, comparado com o valor da PST do solo antes do experimento, este aumento se deve quase que, exclusivamente, ao aumento da salinidade da água de irrigação, com os 
valores indicando sérios riscos de sodicidade (RICHARDS, 1954) ou alta possibilidade de toxicidade causada pelo excesso de sódio no ambiente radicular, exceto quando se irrigou com a água de menor salinidade $\left(\mathrm{CEa}=0,2 \mathrm{dS} \mathrm{\textrm {m } ^ { - 1 }}\right)$. Por outro lado, verifica-se que o aumento na concentração de biofertilizante ou não influenciou ou provocou redução na PST,

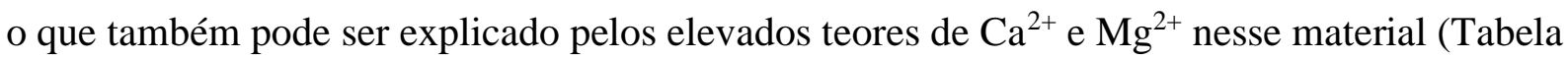
3), pois os menores valores da PST neste trabalho foram evidenciados quando se irrigou com água não salina (Tabela 1). Lacerda et al. (2011) encontraram aumento da porcentagem de sódio trocável no solo irrigado com água salina, provavelmente, devido à composição da água salina utilizada para a irrigação, que apresentava uma proporção maior $\mathrm{de}^{\mathrm{Na}^{+}}$que $\mathrm{Ca}^{2+} \mathrm{e}$ $\mathrm{Mg}^{2+}$ em sua composição. Aumentos da PST do solo com a salinidade foram encontrados por vários autores (WANG et al., 2014; CUCCI et al., 2015).

\section{CONCLUSÕES}

Houve aumento da condutividade elétrica do solo e da PST com o uso de água salina e o biofertilizante. Deve-se ter cautela com a aplicação de biofertilizante concomitante a água salina no cultivo de sorgo pois poderá causar um passivo ambiental.

\section{REFERÊNCIAS}

BADAR, R.; BATOOL, B.; ANSARI, A.; MUSTAFA, S.; AJMAL, A., PERVEEN, S. Amelioration of salt affected soils for cowpea growth by application of organic amendments. Journal of Pharmacognosy and Phytochemistry, v.3, n.6, p. 87-90, 2015.

CAVAlCANTE, L. F.; VIEIRA, M. D. S.; SANTOS, A. D.; OLIVEIRA, W. D.; NASCIMENTO, J. D. Água salina e esterco bovino líquido na formação de mudas de goiabeira cultivar Paluma. Revista Brasileira de Fruticultura, v.32, n.01, p.251-261. 2010.

CUCCI, G.; LACOLLA, G.; PAGLIAI, M.; VIGNOZZI, N. Effect of reclamation on the structure of silty-clay soils irrigated with saline-sodic waters. International Agrophysics, v.29, n.1, p.23-30, 2015.

DIAS, N. S.; COSME, C. R.; SOUZA, A. C. M.; SILVA, M. R. F. Gestão das águas residuárias provenientes da dessalinização da água salobra. In: Recursos hídricos em regiões semiáridas: Estudos e aplicações. GHEYI, H. R.; PAZ, V. P. S.; MEDEIROS, S. S; GALVÃO, C. O (Eds.) - Campina Grande, PB: INSA, Cruz das Almas, BA: UFRB. p.176$187,2012$.

LACERDA, C. F.; SOUSA, G. G.; SILVA, F. L. B.; GUIMARÃES, F. V. A.; SILVA, G. L.; CAVALCANTE, L. F. Soil salinization and maize and cowpea yield in the crop rotation system using saline waters. Engenharia Agrícola, Jaboticabal, v.31, n.4, p.663-675, jul./ago 2011. 
LIMA NETO, A. J.; CAVALCANTE, L. F.; NUNES, J. C.; SOUTO, A. G. L.; BEZERRA, F. T. C. Mudas de tamarindeiro irrigadas com água salina em solo sem e com biofertilizantes. Irriga, Botucatu, v. 20, n. 4, p. 730-744, 2015.

MISHRA, D.J.; SINGH, R.; MISHRA, U. K.; KUMAR, S. S. Role of Bio-Fertilizer in Organic Agriculture: A Review. Research Journal of Recent Sciences, v.2, p.39-41, 2013.

OUNI, Y.; GHNAYA, T.; MONTEMURRO, F.; ABDELLY, C.; LAKHDAR, A. The role of humic substances in mitigating the harmful effects of soil salinity and improve plant productivity. International Journal of Plant Production, v.8, n.3, 2014.

RICHARDS, L. A. Diagnosis and improvement of saline and alkali soils.Washington D.C., U.S. Salinity Laboratory, 1954. 160p. (USDA Agriculture Handbook, 60)

SANTOS, R.V.; CAVALCANTE, L.F.; VITAL, A.F.M. Interações salinidade-fertilidade do solo. In: Manejo da salinidade na agricultura: estudos básicos e aplicados. Instituto Nacional de Ciência e Tecnologia em Salinidade. Fortaleza, CE. parte II, cap 14, p. 221-252. 2010.

VAN HORN, D. J.; OKIE, J. G.; BUELOW, H. N.; GOOSEFF, M. N.; BARRETT, J. E.; TAKACS-VESBACHA, C. D. Soil microbial responses to increased moisture and organic resources along a salinity gradient in a polar desert. Applied and Environmental Microbiology, v.80, n.10, p. 3034-3043, 2014.

WANG, L.; SUN, X.; LI, S.; ZHANG, T.; ZHANG, W.; ZHAI, P. Application of Organic Amendments to a Coastal Saline Soil in North China: Effects on Soil Physical and Chemical Properties and Tree Growth. PLoS ONE, v.9, n.2. 2014. 